\title{
Durability of Hbsag Loss During or After Antiviral Treatment and Impact on Clinical Outcome
}

\author{
${ }^{1}$ Hepatologische Praxis, Germany. \\ ${ }^{2}$ I Medizinische Klinik, Universitätsmedizin Mainz, Germany \\ ${ }^{3}$ Hepatologische Schwerpunktpraxis, Germany \\ ${ }^{5}$ Gastroenterologie am Bayerischen Platz, Germany \\ ${ }^{6}$ Klinik für Gastroenterologie, Hepatologie and Endokrinologie, Medizinische Hochschule Hannover, Germany \\ ${ }^{7}$ Diabetes Zentrum Mergentheim, Theodor-Klotzbücher-Str, Germany \\ ${ }^{8}$ IFI - institute for interdisciplinary medicine, Germany
}

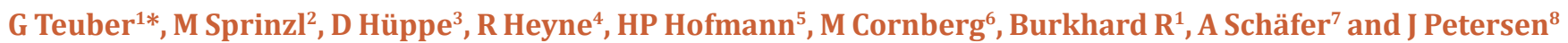

Submission: August 17, 2020; Published: September 10, 2020

*Corresponding author: Gerlinde Teuber, Hepatologische Praxis, Dreieichstr. 59, 60594 Frankfurt/M, Germany

\begin{abstract}
Aims: Concerning the durability of HBsAg loss during or after antiviral treatment only a few studies with conflicting results are available indicating a rate of reactivation with reocurrence of HBsAg and/or HBV-DNA in 4.2\% - 16.4\% in predominantly Asian patients. However, there are no larger European studies available concerning the durability of HBsAg loss during or after antiviral treatment and the impact on further clinical outcome.

Patients and Methods: In this retrospective German multicentre study, 143 patients with chronic hepatitis B (mean age: $43 \pm 13.8$ years, 93 males, 50 females) who lost HBsAg during or after antiviral treatment were included. Antiviral treatment with peginterferon-alpha2a (PegIFNalpha2a) and/or nucleus(t)ides were administered between April 2008 and July 2014. Before antiviral treatment, 17 patients had established liver cirrhosis. Primary endpoint was reactivation with reoccurrence of HBsAg Further endpoints were clinical progressive liver disease, liver transplantation and death.

Result: During the follow-up period (mean: $3.0 \pm 2.1$ years) a reoccurrence of HBsAg was observed in only 3/143 patients (2.1\%), and in none with previous seroconversion to Anti-HBs. The HBV reactivation in these patients was not associated with detectable HBV-DNA levels, $2 / 3$ patients had baseline cirrhosis and $1 / 2$ subsequently died due to recurrent multifocal hepatocellular carcinoma. Among the 140 patients with persisting HBsAg loss, two initially cirrhotic patients died, and one received liver transplantation all due to hepatocellular carcinoma.

Conclusion: In a predominantly Caucasian patient population HBsAg loss during and/or after antiviral treatment seems to be durable with low rates of reactivation. Cirrhotic patients, however, have a high risk developing hepatocellular carcinoma even after HBsAg loss. Continous
\end{abstract} surveillance in these patients seems to be mandatory.

Keywords: Clinical outcome, HBsAg, Viral suppression, Hepatocellular carcinoma

\section{Introduction}

Globally, approximately 257 million people are affected by chronic hepatitis B virus (HBV) infection, a major cause of liver cirrhosis, portal hypertension, liver failure, hepatocellular carcinoma and death [1-5]. In the chronic sequalae the development of liver cirrhosis and hepatocellular carcinoma, are strongly dependent on HBV viral load [6,7]. An effective antiviral treatment with sustained viral suppression can prevent progression to liver cirrhosis with its potential lethal complications $[8,9]$. Furthermore, long term antiviral treatment has been shown to reduce the degree of inflammation and fibrosis [10,11]. In Germany, two different treatment approaches are available, one with pegylated Interferon-alpha2a (pegIFN-alpha2a), combining 
immunomodulatory and direct antiviral activity and one with direct antiviral acting nucleosides and nucleotides [12,13]. An immunologic control of the disease with loss of HBsAg can be achieved by a one-year course of pegIFN-alpha2a in $3-5 \%$ of the patients after a 24 weeks follow-up period $[14,15]$. The rate of HBsAg loss can increase up to $12 \%$ in these patients after a 5 years long term follow-up [16]. During long-term treatment with nucleoside(t)es HBsAg clearance can be achieved in $11.8 \%$ of the patients with chronic HBV after a 7 years treatment period [17]. Concerning the durability of HBsAg loss during or after antiviral treatment, especially with nucleoside(t)es, only a few studies with conflicting results are available indicating a rate of reactivation with reoccurrence of HBsAg and/or HBV-DNA in 4.2\% - 16.4\% [18-20] in predominantly Asian patients. Up to now, there are no data from European patients available concerning the durability of HBsAg loss during or after antiviral treatment and the impact on further clinical outcome. Aim of the present study was to assess the durability of HBsAg loss and seroconversion to anti-HBs during and after antiviral treatment with pegIFN-alpha2a and/ or nucleoside(t)es in a European patient collective. Secondary endpoint was the clinical impact of HBsAg loss on patient's longterm outcome, determined by progressive liver disease, liver transplantation and death.

\section{Patients and Methods}

\section{Study design}

In this large retrospective German multicentre study, patients with chronic hepatitis B who lost HBsAg during or after antiviral treatment with peginterferon-alpha (PegIFN-alpha) and/or nucleus(t)ides between April 2008 and July 2014 were eligible. Inclusion criteria were chronic hepatitis $B$ with persistence of HBsAg for at least 6 months and/or sonographic, radiologic or histologic evidence of chronic liver disease and age>18 years before the begin of first antiviral treatment. Exclusion criteria were coinfection with hepatitis D virus, hepatitis C virus, and human immunodeficiency virus-coinfection as well as the presence of other concomitant liver diseases, i.e. autoimmune hepatitis/ cholangitis, primary hemochromatosis, Morbus Wilson and alpha1-antitrypsin deficiency) and previous liver transplantation. During antiviral treatment clinical examinations were routinely performed every 3-6 months, including determination of transaminases, liver function tests, and HBV-DNA. After cessation of antiviral treatment controls were performed every 6-12 months. In addition, ultrasound examination and determination of HBsAg and Anti-HBs were performed during and after antiviral treatment every 6-12 months as clinically indicated. Primary endpoint was to assess the frequency of reactivation with reoccurrence of HBsAg. Secondary clinical endpoints were to identify risk factors for HBV-reactivation, and to assess the occurrence of liver cirrhosis, hepatic decompensation, hepatocellular carcinoma, liver transplantation, and death. The study protocol was reviewed and approved by the local ethic commission of Landesärztekammer Hessen. With respect to the retrospective character of the present study and the pseudonymous data collection informed consent of the participating individuals was not required.

\section{Patients}

In the participating seven German centers 143 patients B (mean age: $43+13.8$ years, 93 males, 50 females) were identified who met the respective in- and exclusion criteria. The patients received antiviral treatment with peginterferon-alpha2a (PegIFNalpha2a) and/or nucleus(t)ides between April 2008 and July 2014. Demographic and clinical characteristics of the study population are shown in Table 1. Prior to antiviral treatment, eight patients developed an acute flair of chronic hepatitis B virus infection. Liver cirrhosis was already present in 17 CHB-patients and two of these patients had hepatocellular carcinoma receiving partial liver resection before antiviral treatment.

Table 1: Demographic and clinical characteristics of the $143 \mathrm{CHB}$ patients with HBsAg loss before antiviral treatment.

\begin{tabular}{|c|c|}
\hline & CHB-Patients \\
\hline & $(n=143)$ \\
\hline \multirow{2}{*}{ Age (years)* } & $43.2+13.8$ \\
\hline & $(18.0-78)$ \\
\hline \multicolumn{2}{|c|}{ Sex } \\
\hline male & $93(65 \%)$ \\
\hline female & $50(35 \%)$ \\
\hline \multirow{2}{*}{ disease duration: } & $53.1+59.6$ \\
\hline & $(6.0-271)$ \\
\hline \multicolumn{2}{|c|}{ Mode of Transmission } \\
\hline perinatal & 27 (18.9\%) \\
\hline household contacts & 27 (18.9\%) \\
\hline sexual & $20(14,0 \%)$ \\
\hline IVDA & $12(8.4 \%)$ \\
\hline medical procedures & $2(1.4 \%)$ \\
\hline Unknown & $74(51.7 \%)$ \\
\hline \multicolumn{2}{|c|}{ Region of Origin } \\
\hline Europe & $80(55,9 \%)$ \\
\hline Asia & $59(41.3 \%)$ \\
\hline Other & $4(2.8 \%)$ \\
\hline liver cirrhosis & $17(11.9 \%)$ \\
\hline ascites & $3(2.1 \%)$ \\
\hline esophageal varices & $9(6.3 \%)$ \\
\hline episode of bleeding & $1(0.7 \%)$ \\
\hline hepatocellular carcinoma & $2(1.4 \%)$ \\
\hline
\end{tabular}

*Results are given as mean + SD (minimum-maximum). IVDA: intravenous drug abuse. 


\section{Statistical analysis}

Data were collected before the begin of antiviral treatment, at the timepoint of HBsAg loss and from the latest available follow up examination. Due to the low rate of HBsAg reactivation statistical analysis was performed descriptively using Stat view version 5.0 1999. Data are shown as mean + SD.

Results

\section{Antiviral treatment}

Patients received an antiviral treatment with pegIFN-alpha2a only, nucleus(t)ides as monotherapy, sequential therapy or as a combined treatment approach. The antiviral treatment was monitored from 2000-2015. Antiviral therapy was based on approved drugs, which were chosen by the participating centers according to the German hepatitis B treatment guidelines at the given timepoint of treatment initiation. 37 patients received more than one antiviral treatment until HBsAg loss was achieved (Table 2). Main reasons for treatment switch were the development of viral resistance and insufficient viral suppression, especially in patients treated with lamivudine or adefovir dipivoxil. In patients receiving pegIFN-alpha2a side effects were the main reason for treatment cessation. The average treatment duration until loss of HBsAg were 50 weeks for patients receiving pegIFN-alpha2a monotherapy, 290 weeks in the patients receiving nucleus(t) ides, and 201 weeks in patients treated with a combination of pegIFN-alpha2a and nucleus(t)ides. After loss, of HBsAg antiviral treatment was continued according to the type of antiviral treatment for 24 weeks (pegIFN-alpha2a only), 44 weeks (NUCs only) and 39 weeks (NUCs combined with pegIFN-alpha2a).

Table 2: Antiviral treatment.

\begin{tabular}{|c|c|c|}
\hline & 1. Antiviral Treatment (n=143) & >1 Antiviral Treatment (n=37) \\
\hline pegIFN-alpha2a & $41(28.7 \%)$ & $2(5,1 \%)$ \\
\hline LAM & $32(22.4 \%)$ & $2(5,4 \%)$ \\
\hline ADV & $3(2.1 \%)$ & $9(24,3 \%)$ \\
\hline ETV & $24(16.8 \%)$ & $14(37,8 \%)$ \\
\hline TDF & $39(27.3 \%)$ & $4(10,8 \%)$ \\
\hline ADV/ETC & $2(1.4 \%)$ & -- \\
\hline ETV/TDF & --- & $1(2,7 \%)$ \\
\hline ETV/pegIFN-alpha2a & --- & $2(5,4 \%)$ \\
\hline TDF/pegIFN-alpha2a & $1(0.7 \%)$ & --- \\
\hline TDF/ETV/pegIFN-alpha2a & $1(0.7 \%)$ & \\
\hline
\end{tabular}

PegIFN-alpha2a: pegylated Interferone-alpha2a, LAM: Lamivudine, ADV: Adefovir Dipivoxil, ETV: Entecavir, TDF: Tenofovir Disoproxil Fumarate

\section{Virologic and biochemical outcome}

The mean follow up period after HBsAg loss was $3.0+2.1$ years. At the timepoint of loss of HBsAg only 35 patients had simultaneously a detectable Anti-HBs (Table $3 \& 4$ ) The number of Anti-HBs positive patients increased to $n=50$ after the end of the follow-up period. At the time point of HBsAg loss qualitative HBVDNA was still detectable in 8 patients (quantifiable in 5 patients: mean $273 \times 103 \mathrm{IU} / \mathrm{mL}$ ). This was associated with a slight ALT- elevation $<1,5$ ULN in three patients and a marked ALT-elevation in one patient. After the end of follow up qualitative HBV-DNA was still positive in 5/140 of the patients with sustained HBsAg loss, but quantifiable only in 1 patient (HBV-DNA 680x103 IU/mL). Only this patient had also a slightly elevated ALT. At the time point of HBsAg loss 31 patients had elevated ALT-levels, and 20 patients had still elevated ALT-Levels at the end of follow-up, which was associated with detectable HBV-DNA in only one of these patients.

Table 3: Treatment duration according to the type of antiviral treatment.

\begin{tabular}{|c|c|c|c|}
\hline & PegIFN-alpha2a & NUCs & PegIFN-alpha2a/ \\
\hline $\mathrm{n}=37$ & $\mathrm{n}=93$ & $\mathrm{n}=13$ & $201+189$ \\
\hline Total treatment & $50+7$ & $290+193$ & $(201-677)$ \\
\hline duration* & $(20-78)$ & $(21-776)$ & $237+220$ \\
\hline Treatment duration & $38+12$ & $234+183$ & $(12-665)$ \\
\hline until loss of HBsAg* & $(12-52)$ & $(8-730)$ & $39+29$ \\
\hline Treatment duration & $24+24$ & $44+25$ & $(12-100)$ \\
\hline After loss of HBsAg* & $(8-130)$ & $(4-114)$ & \\
\hline
\end{tabular}

${ }^{*}$ Results are given as mean + SD (minimum-maximum). 
Table 4: Clinical outcome at the time of HBsAg loss and at the end of follow-up ( $n=143$ patients).

\begin{tabular}{|c|c|c|}
\hline & HBsAg loss & End of Follow-up \\
\hline \multirow{2}{*}{ ALT (IU/L)* } & $43+38$ & $(5-107)$ \\
\hline \multirow{2}{*}{ Bilirubine (mmol/L)* } & $(9-326)$ & $13.7+6.8$ \\
\hline \multirow{2}{*}{ HBsAg: negative } & $15.4+8.6$ & $(3.4-39.3)$ \\
\cline { 2 - 3 } & $(3.4-44.5)$ & 3 \\
\hline Anti-HBs: positive & 143 & 50 \\
\hline HBV-DNA: detectable & 0 & 5 \\
\hline Cirrhosis & 35 & 13 \\
\hline Hepatocellular carcinoma & 8 & 4 \\
\hline Liver transplantation & 17 & 1 \\
\hline Death+ & -- & 5 \\
\hline
\end{tabular}

*Data are given as mean + standard deviation (minimum-maximum).

+Three death were due to mutlifocal hepatocellular carinoma and two cases were not related to chronic liver disease.

Reoccurrence of HBsAg was observed in only 3/143 (2.1\%) patients, HBV-reactivation occured in one patient 6 years and in 2 patients 10 years after HBsAg loss. HBV reactivation was not associated with detectable HBV-DNA levels in all of the three patients. However, one of these patients had slightly elevated transaminases. Two out of these three patients received a combination of a nucleos(t)ide and pegIFN-alpha2a and the third patient a treatment with various nucleon(s)ides.

\section{Clinical outcome}

Among the 140 patients with sustained HBsAg loss only 15/140 had liver cirrhosis prior to treatment. Except of one patient with child-Turcotte Pugh (CTP) class B all other cirrhotic HBV-patients were compensated. During the follow-up period in two of the cirrhotic patients' multifocal hepatocellular carcinoma was diagnosed 3 years and 5 years after HBsAg clearance. Both patients died subsequently. Another cirrhotic patient developed hepatocellular carinoma 7 years after HBsAg clearance and received curative orthotopic liver transplantation One of these patients received lamivudine treatment and two patients were treated with pegIFN-alpha2a only. Further two patients died due to non-HBV-related causes (gastric cancer and myocardial infarction). Hydropic decompensation and hepatic failure were only observed in the two patients who died due to progressive hepatocellular carinoma. In addition, one of these patients had a gastrointestinal bleeding due to esophageal varices. Two out of three patients with HBV reactivation had liver cirrhosis, both CTP class A. One cirrhotic patient initially received lamivudine. After two years he was switched to Adefovir Dipivoxil due to clinically suspected lamivudine-resistance. After 4 years of ADV-treatment the patient lost HBsAg. However. HBV-DNA ( 2.1 x $103 \mathrm{IU} / \mathrm{ml}$ ) was still detectable in this patient. Entecavir was added to the Adefovir
Dipivoxil treatment. But however, in parallel to the HBsAg loss non respectable hepatocellular carcinoma was diagnosed. The patient received chemoembolization and died four years later due to progressive hepatocellular carcinoma and hepatic failure. However, a few days before his death a reactivation of hepatitis $B$ with recurrence of HBsAg could be observed but still without detectable HBV-DNA.

\section{Discussion}

In this large retrospective study, we could clearly show that the loss of HBsAg during or after antiviral treatment was durable in most of our predominantly European cohort with a low rate of reactivation of only $2.1 \%$ during the 3 years long-term follow-up period. The rate of reactivation was markedly higher in cirrhotic than in non-cirrhotic patients (11.8 vs. $0.7 \%$ ). Despite sustained HBsAg loss, cirrhotic patients had a persisting high risk for hepatocellular carcinoma (23.5\%) while non-cirrhotic patients had no evidence for disease progression or hepatocellular carcinoma. So far, the largest published study comparing the durability of HBsAg loss in untreated and nucleus(t)ide treated Chinese patients confirmed our results of a low reactivation rate and a favorable clinical outcome after HBsAg clearance [18]. The incidence of hepatocellular carcinoma and liver related death was $0.3 \%$, and $1.3 \%$ respectively. Hepatocellular carcinoma occurred more frequently in patients with HBsAg stereovision (14.3\%). However, in this study detailed clinical data concerning the cirrhosis status, hydropic decompensation and variceal bleeding are not available. A recently published study in Korean patients showed a higher cumulative reactivation rate of $11.7 \%$ which was transient in most patients [20]. Compared to our data, the higher reactivation rate in Asian patients may be explained by a longer disease duration following vertical transmission and by a higher 
percentage of patients with liver cirrhosis. HBsAg reversion overserved in three cases was permanent in two of our patients whereas one patient died a few days after HBsAg reoccurrence due to hepatocellular carinoma. In concordance with our data sustained HBsAg loss was associated with a favorable clinical outcome while cirrhotic patients were still at risk for developing hepatocellular carinoma or liver failure. These findings are confirmed by other previously published studies concerning the clinical long-term course after spontaneous HBsAg clearance reporting an association between HBsAg clearance and a reduced risk of disease progression and hepatocellular carcinoma compared to patients without HBsAg loss [21-23]. Previously published smaller studies indicated a higher risk for HBVreactivation in patients treated with nucleus(t)ides compared to patients treated with interferon-alpha [24,25]. These findings are not confirmed by our study collective with respect to the limited number of HBsAg reoccurrence. The majority of our patients received an antiviral treatment with nucleus(t)ides only. Of the patients with HBsAg reoccurrence two out of three patients received a therapy with nucleus(t)ides combined with pegIFNalpha2a. Thus, antiviral treatment with pegIFN-alpha seems not to be superior to a treatment with nucleus(t)ides in terms of HBVreactivation.

Furthermore, most of these smaller studies investigated the durability of HBsAg loss after lamivudine treatment, a drug with a known high rate of viral resistance and virologic breakthrough during antiviral treatment $[26,27]$. Since most of our patients were initially treated with or switched to a second generation drug providing a high barrier of resistance, i.e. entecavir or tenofovir disoproxil fumarate, HBV-reactivation with reoccurrence of HBsAg was observed only in $0.7 \%$ of our patients treated with nucleus $(\mathrm{t})$ ides only compared to $4 \%$ of the patients receiving pegIFNalpha2a only or combined with nucleus(t)ides. Thus, the data of the present study indicate that in terms of posttreatment HBVreactivation, pegIFN-alpha2a treatment is not superior compared to a treatment with highly effective second-generation nucleus $(\mathrm{t})$ ides. With respect to the fact that only five patients with sustained HBsAg loss had detectable HBV-DNA at the end of follow-up, there was also no evidence for the development of escape mutants. However, sequencing of HBV-DNA to exclude the presence of mutants not expressing HBsAg was not available in these patients. The present study has several limitations. One mayor limitation is the retrospective approach. However, prospective studies investigating the long-term durability of HBsAg are currently not available. The lack of prospective clinical trials is explained by the low rate of HBsAg loss during or after antiviral treatment and the long treatment durations in NUC patients. Thus, we were able to identify in the participating seven large German hepatology centers only 143 HBV-patients with HBsAg loss during or after antiviral treatment within a 6 years' time period. Since the data include all of the available CHB-patients by the participating centers there was no bias for patient selection. Another limitation of the present study is the variation in antiviral regimens used in our study population. However, this is a reflection of a German real-life setting considering the avaibility of different drugs and the changing German guidelines in the last two decades. Thus, even in this large German real-life cohort of HBV-patients HBsAg loss was durable, irrespective of the used antiviral drug. In conclusion the study clearly demonstrate that HBsAg loss during and/or after antiviral treatment is durable with low rates of offtreatment reactivations in a predominantly Caucasian patient population. However, cirrhotic patients seems to have higher risk for HBV-reactivation Despite sustained HBsAg loss cirrhotic patients have still a substantial risk for developing hepatocellular carinoma. Thus, regular HBsAg monitoring and long-term HCCsurveillance of these patients should be mandatory.

\section{Acknowledgement}

This publication contains data of the doctoral thesis of Cand Med Rita Burkhard and Malte Kleinert. This study was financially supported by Gilead Sciences.

\section{References}

1. WHO (2017) Global Hepatitis Report 2017. Geneva: World Health Organization.

2. Lok AS, McMahon BJ (2007) Chronic hepatitis B. Hepatology 45(2): 507-439.

3. Ganem D, Prince AM (2004) Hepatitis B virus infection-natural history and clinical consequences. N Engl J Med 350(11): 1118-1129.

4. Fattovich G (2003) Natural history and prognosis of hepatitis B. Semin Liver Dis 23(1): 47-58.

5. Fattovich G, Stroffolini T, Zagni I, Donato F (2004) Hepatocellular carcinoma in cirrhosis: incidence and risk factors. Gastroenterology 127: S35.

6. Iloeje UH, Yang HI, Su J, Jen CL, You SL, et al. (2006) Risk Evaluation of Viral Load Elevation and Associated Liver Disease/Cancer-In HBV (the REVEAL-HBV) Study Group. Predicting cirrhosis risk based on the level of circulating hepatitis B viral load. Gastroenterology 130(3): 678-686.

7. Chen CJ, Yang HI, Su J, Jen CL, You SL, et al. (2006) REVEAL-HBV Study Group. Risk of hepatocellular carcinoma across a biological gradient of serum hepatitis B virus DNA level. JAMA 295(1): 65-73.

8. Papatheodoridis GV, Chan HL, Hansen BE, Janssen HL, Lampertico P (2015) Risk of hepatocellular carcinoma in chronic hepatitis B: Assessment and modification with current antiviral therapy. J Hepatol 62: 956-967.

9. Su TH, Hu TH, Chen CY, Huang YH, Chuang WL, et al. (2016) C-TEAM study group and the Taiwan Liver Diseases Consortium. Four-year entecavir therapy reduces hepatocellular carcinoma, cirrhotic events and mortality in chronic hepatitis B patients. Liver Int 36: 1755-1764.

10. Marcellin P, Gane E, Buti M, Afdhal N, Sievert W, et al. (2013) Regression of cirrhosis during treatment with tenofovir disoproxil fumarate for chronic hepatitis B: a 5-year open-label follow-up study. Lancet 381 : 468-475.

11. Papachrysos N, Hytiroglou P, Papalavrentios L, Sinakos E, Kouvelis I, et al. (2015) Antiviral therapy leads to histological improvement of HBeAg-negative chronic hepatitis B patients. Ann Gastroenterol 28(3): 374-378. 
12. M Cornberg, U Protzer, MM Dollinger, J Petersen, H Wedemeyer, et al. (2007) Prophylaxis, Diagnosis and Therapy of Hepatitis-B-Virus (HBV)Infection: upgrade of the guideline, AWMF-Register 021/011. Z Gastroenterol 45: 525-574.

13. M Cornberg, U Protzer, J Petersen, H Wedemeyer, T Berg, et al. (2011) Prophylaxis, Diagnosis and Therapy of Hepatitis B Virus Infection- The German Guideline. AWMF-Register-Nr. Z Gastroenterol 49: 871-930.

14. Janssen HL, van Zonneveld M, Senturk H, Zeuzem S, Akarca US, et al. (2005) HBV 99-01 Study Group; Rotterdam Foundation for Liver Research. Pegylated interferon alfa-2b alone or in combination with lamivudine for HBeAg-positive chronic hepatitis B: a randomised trial. Lancet 365(9454): 123-129.

15. Lau GK, Piratvisuth T, Luo KX, Marcellin P, Thongsawat S, et al. (2005) Peginterferon Alfa-2a HBeAg-Positive Chronic Hepatitis B Study Group. Peginterferon Alfa-2a, lamivudine, and the combination for HBeAg-positive chronic hepatitis B. N Engl J Med 352(26): 2682-2695.

16. Marcellin P, Piratvisuth T, Brunetto M (2009) 924 Increasing rates of HBsAg clearance and seroconversion in patients with HBeAg-negative disease treated with peginterferon alfa-2a \pm lamivudine: results of 5-year post-treatment follow up. J Hepatol 50: S336.

17. Buti M, Tsai N, Petersen J, Flisiak R, et al. (2014) Seven-year efficacy and safety of treatment with tenofovir disoproxil fumarate for chronic hepatitis B virus infection. Digestive diseases and sciences 60(5): 1457-1464.

18. Yip TC, Wong GL, Wong VW, Tse YK, Lui GC, et al. (2018) Durability of hepatitis B surface antigen seroclearance in untreated and nucleos $(\mathrm{t})$ ide analogue-treated patients. J Hepatol 68: 63-72.
19. Chi H, Wong D, Peng J, Cao J, Van Hees S, et al. (2016) Clinical outcomes after spontaneous and nucleos(t)ide analogue treated HBsAg seroclearance in chronic HBV infection. Aliment Pharmacol Ther 43(12): 1311-1318.

20. Kim GA, Lim YS, An J, Lee D, Shim JH, et al. (2014) HBsAg seroclearance after nucleoside analogue therapy in patients with chronic hepatitis B: clinical outcomes and durability. Gut 63(8): 1325-1332.

21. Chen YC, Sheen IS, Chu CM, Liaw YF (2002) Prognosis following spontaneous HBsAg seroclearance in chronic hepatitis B patients with or without concurrent infection. Gastroenterology 123(4): 1084-1089.

22. Ahn SH, Park YN, Park JY, Chang HY, Lee JM (2005) Long-term clinical and histological outcomes in patients with spontaneous hepatitis B surface antigen seroclearance. J Hepatol 42(2): 188-194.

23. Liu J, Yang HI, Lee MH, Lu SN, Jen CL et al. (2014) Spontaneous seroclearance of hepatitis B seromarkers and subsequent risk of hepatocellular carcinoma. Gut 63(10): 1648-1657.

24. Lau GK (2009) HBeAg-positive chronic hepatitis B: why do I treat my patients with pegylated interferon. Liver Int 29 Suppl 1: 125-129.

25.Wu D, Ning Q (2017) Toward a Cure for Hepatitis B Virus Infection: Combination Therapy Involving Viral Suppression and Immune Modulation and Long-term Outcome. J Infect Dis 216(8): S771-S777.

26. Hsu CW, Yeh CT, Chang ML, Liaw YF (2007) Identification of a hepatitis $B$ virus $\mathrm{S}$ gene mutant in lamivudine-treated patients experiencing HBsAg seroclearance. Gastroenterology 132: 543-550.

27. Yeh CT (2010) Development of HBV S gene mutants in chronic hepatitis $\mathrm{B}$ patients receiving nucleotide/nucleoside analogue therapy. Antivir Ther 15: 471-475. 